\title{
APROXIMACIONES A LA CULTURA DIGITAL DE LAS FAMILIAS VENEZOLANAS EN TORNO AL PROYECTO CANAIMA EDUCATIVO'
}

Yaimar del Valle Montoya GonzÁlez ${ }^{2}$

Universidade Federal Da Bahia, Brasil / ymontoyag@gmail.com

María Helena BONILLA ${ }^{3}$

Universidade Federal Da Bahia, Brasil / bonillabr@gmail.com

Recibido: 30/3/2019/ Aceptado: 7/8/2019

doi: 10.26439/contratexto2019.n032.4620

Resumen. El proyecto Canaima Educativo incorporó computadoras portátiles (Canaimitas) en los espacios educativos públicos de Venezuela y, aunque entre sus propuestas estuvo la integración de las familias, esta política no fue explícita para su desarrollo. Nuestro objetivo fue interpretar los usos que las familias atribuyen a la computadora Canaimita, entendiendo que es necesario incluir las tecnologías digitales en la cotidianidad, ya que vivimos en una sociedad inmersa en la cultura digital. Por lo tanto, las políticas públicas deben proporcionar acompañamiento desde una perspectiva amplia e integradora. La metodología fue cualitativa con enfoque hermenéutico, de inspiración etnográfica. El trabajo de campo fue realizado en el estado Vargas, en dos instituciones educativas de secundaria; los cuatro estudiantes y sus grupos familiares participantes fueron seleccionados por medio de cuestionarios online, con los cuales realizamos grupos focales. Las informaciones fueron organizadas a través del análisis textual discursivo, con apoyo del software Atlas.ti. Los resultados de la investigación demuestran la falta de acompañamiento para integrar las Canaimitas en los hogares, por lo que las familias se aproximan a la constitución de la cultura digital desde cada una de sus generaciones con prácticas espontáneas y dinámicas, de acuerdo con intereses propios.

Palabras clave: Canaima Educativo / cultura digital / familias / políticas públicas

1 Este artículo se desarrolla a partir del trabajo de grado, para la obtención del título de magíster en Educación titulado: Proyecto Canaima Educativo y la cultura digital en las familias venezolanas defendida en la Universidade Federal da Bahia (2018). El presente trabajo fue realizado con apoyo de la Coordinación de Perfeccionamiento de Personal de Nivel Superior - Brasil (CAPES) - Código de Financiamiento 001.

2 Doctoranda en Educación por la Universidade Federal da Bahia, Brasil. http://orcid.org/0000-0003-1870-9678 3 Doctora en Educación por la Universidade Federal da Bahia, Brasil. https://orcid.org/0000-0002-0137-6363 


\title{
VENEZUELAN FAMILIES' APPROACHES TO DIGITAL CULTURE AROUND THE CANAIMA EDUCATIVO PROJECT
}

\begin{abstract}
The Canaima Educativo project supplied laptops (canaimitas) to Venezuela's public schools. Although family integration was among the project's objectives, there were not guidelines on how to achieve such objective. This research aimed to interpret how families used the canaimitas considering the importance to include digital technologies in daily life, since our society is living in a digital culture. Therefore, public policies should provide support from a broad and inclusive perspective. We used an ethnographic qualitative methodology with a hermeneutic approach. Fieldwork was performed at two high schools located in the state of Vargas. Four (4) students and their families were selected using online surveys and participated in focus group interviews. The information was organized through a textual discourse analysis using the ATLAS.ti software. The research results showed the lack of support to include the canaimitas to those families' homes. In this sense, the members of such families approach to the digital culture with spontaneous and dynamic practices according to their own interests.
\end{abstract}

Keywords: Canaima Educativo, digital culture, families, public policies

\section{APROXIMAÇÕES À CULTURA DIGITAL DAS FAMÍLIAS VENEZUELANAS EM TORNO AO PROJETO CANAIMA EDUCATIVO}

Resumo. 0 projeto Canaima Educativo incorporou computadores portáteis (Canaimitas) nos espaços educativos públicos na Venezuela e, embora tenha aparecido como objetivo a integração das famílias, essa política não foi perceptível no seu desenvolvimento. Nessa linha, nosso objetivo foi interpretar as funções que as famílias atribuem ao computador "Canaimita", entendendo que é preciso incluir as tecnologias digitais em nossa cotidianidade, pois vivemos em uma sociedade mergulhada na cultura digital. É por isso que as políticas públicas devem fornecer acompanhamento a partir de uma perspectiva ampla e inclusiva. A metodologia foi de natureza qualitativa com um enfoque hermenêutico, de inspiração etnográfica. 0 trabalho de campo foi realizado no estado Vargas, em duas instituições educativas de ensino médio; os quatro estudantes e seus grupos familiares participantes foram selecionados por meio de questionários online, com o qual conduzimos grupos focais e as informações foram organizadas através da análise textual discursiva, com apoio do software Atlas.ti. Os resultados da pesquisa mostram a falta de acompanhamento para integrar as Canaimitas nos lares, motivo pelo qual as famílias se aproximaram da constituição da cultura digital a partir do desenvolvimento de práticas espontâneas e dinâmicas de acordo com interesses próprios, acordes com suas gerações.

Palavras chave: Canaima Educativo, Cultura digital, Famílias, Políticas Públicas. 


\section{INTRODUCCIÓN}

El desarrollo de nuestra capacidad de adaptación posibilitó el desenvolvimiento de la forma en que tratamos la información, pasamos del mundo analógico al digital, lo que es denominado proceso de digitalización de la información. Esto hace referencia al sistema binario, un conjunto de cero (0) y uno (1), es decir, la partícula de información más pequeña que contiene una computadora. La digitalización propició la traducción de textos, imágenes y sonidos en bits, y facilitó la manera de obtener, organizar, almacenar y transportar grandes volúmenes de informaciones.

Aunado a esto, también produjo otros modos de comunicarnos, de pensar, de aprender, de relacionarnos, así como de ser y de estar en el mundo. Provocó que haya mudado exponencialmente la manera en que construimos conocimientos y abrió caminos para que otras articulaciones culturales fueran posibles. Por tanto, es necesario comprender que no solo lo digital se adentró en nuestra contemporaneidad, sino que aspectos cognitivos, existenciales, así como experienciales (Lemos, 2015) reconfiguraron nuestras prácticas y actitudes frente al mundo, ya no son las mismas del pasado ni serán las mismas del futuro, porque estamos inmersos en un sistema altamente cambiante, dinámico (Capra, 1996), fluido (Bauman, 2003), estamos viviendo en la cultura digital.

Observamos que en la cultura de la contemporaneidad estamos envueltos de dispositivos digitales que se nos presentan cada día más reducidos en tamaño porque los miniaturizamos o los creamos más portátiles, también más híbridos y ubicuos. Esto refleja la principal característica de las tecnologías digitales, como es la movilidad (Lemos, 2009). Esta particularidad, junto con la conexión en red por medio del wireless, abrió la posibilidad de una nueva estructura comunicativa y organizativa donde existen flujos de corrientes con informaciones constantes, la denominada por Manuel Castell (1999) sociedad en red, donde se redefinieron los usos de las tecnologías digitales y se establecieron otras prácticas sociales.

De ahí que las políticas educativas han intentado incorporar exigencias contemporáneas implantando proyectos que introducen tecnologías de la información y de la comunicación (TIC) en las aulas de clases. En el caso de Venezuela, la creación e incorporación fue a través del proyecto Canaima Educativo, en el año 2009, que integra computadoras portátiles de bajo costo (Canaimitas) a los diferentes niveles educativos públicos del país. A pesar de que este proyecto trascendió las salas de clases y se extendió hasta llegar al ámbito familiar con unos objetivos planteados desde la política educativa, la estrategia de llevar la Canaimita a los hogares de los estudiantes carece de una explicación detallada en cuanto a la manera en que el proceso de integración, formación y acompañamiento acontecería. Es así que nuestro objetivo es interpretar los usos que las familias atribuyen a la computadora Canaima, cuando este dispositivo digital portátil llega a las casas sin una estrategia determinada. 


\section{METODOLOGÍA}

Para aproximarnos a esas interpretaciones, adoptamos un abordaje cualitativo con enfoque hermenéutico ya que nos centramos en la interpretación, así como en la comprensión de las actuaciones, actividades e intercambios entre los sujetos sociales participantes dentro de su cotidianidad. El propósito fue acercarnos a su visión sobre el fenómeno en estudio, concediéndoles valor a sus vivencias, percepciones y emociones como parte esencial de la investigación (Macedo, Assis, Guerra, Rangel y Macedo de Sá, 2014).

Además, hicimos uso de la inspiración etnográfica como una estrategia metodológica que nos auxilió en la obtención de información empírica del campo de estudio. El proyecto se desarrolló en el estado Vargas, Venezuela, entre los meses de mayo y julio del año 2017, en dos unidades educativas en el nivel de secundario, tomando como criterio de escogencia que hayan recibido las computadoras del proyecto Canaima Educativo. Los participantes estuvieron compuestos por cuatro estudiantes y sus núcleos familiares seleccionados a partir de un cuestionario online, que atendieron a los siguientes criterios: aceptar participar en la investigación, ser adolescentes de 15 a 17 años, utilizar la computadora Canaimita en casa, tener acceso a internet en casa y acceso a smartphone, pertenecer a un núcleo familiar de más de dos personas y con seis personas como máximo, tener la mayor cantidad de familiares que desarrollen actividades en la portátil Canaimita y con otras tecnologías digitales.

Para la comprensión de lo que sucede en casa, es decir, las dinámicas que se desenvuelven con la Canaimita, fue necesario elaborar múltiples dispositivos de recolección de informaciones, entre ellos la observación, el principal de la investigación (Balcázar, González, Gurrola y Chimal, 2006). Para registrar todo el proceso de interacción utilizamos un diario de campo, para asentar no solo las informaciones verbales y no verbales de sujetos participantes, sino también para permitirnos, como investigadoras, reafirmar nuestro estatus de actor/autor comprendiendo que nuestro imaginario está evidentemente implicado (Macedo, 2010).

También las familias seleccionadas participaron en un grupo focal, estrategia que se realizó con apoyo de un guion de preguntas previamente elaboradas, con el objetivo de obtener percepciones, opiniones, experiencias, saberes detallados de un tema en específico (Balcázar, González, Gurrola y Chimal, 2006), en este caso sobre el proyecto Canaima Educativo dentro del hogar. Los grupos focales fueron realizados en las casas de las familias participantes, con duración variable para cada una, fueron registrados en audio, fotografía y aprobados por sus rúbricas a través de un consentimiento informado. Es importante destacar que durante todo el proceso se resguardaron las identidades de los participantes, por ello hacemos uso de nombres indígenas venezolanos para referirnos a cada uno de ellos. 
Para alcanzar nuestro objetivo de "interpretar los usos que las familias atribuyen a la computadora Canaimita", trabajamos bajo la perspectiva del análisis textual discursivo. Para Roque Moraes y Maria do Carmo Galiazzi (2016), el análisis textual discursivo es una metodología de análisis que pretende ser un movimiento de autoorganización constante, de deconstrucciones, reconstrucciones y de comprensiones, a partir de la valorización del lenguaje como herramienta de expresión de los sujetos participantes, percibiéndolos, conforme expresa Roberto Sidney Macedo (2010), como autores de su propia realidad. Moraes y Galiazzi (2016) exponen que después de aplicados los instrumentos de recolección de informaciones y tener transcritas las informaciones, se procede a establecer un ciclo de análisis compuesto por el desmontaje de los textos, el establecimiento de relaciones y la captación del nuevo emergente, acompañados de un constante proceso autoorganizado.

El primer foco, el desmontaje del texto, es "un proceso que produce desorden a partir de un conjunto de textos ordenados" (p. 43, traducción nuestra), es decir, de las producciones textuales transcritas, se realiza un ejercicio de desconstrucción y fragmentación, para crear unidades de análisis significativas para el fenómeno estudiado. El segundo elemento del ciclo es el establecimiento de relaciones, que consiste en agrupar las unidades de análisis significativas en categorías (iniciales, intermedias o finales) que pueden contener subcategorías y, a su vez, hacer conexiones entre estas y la teoría; en el caso de este estudio se realizó de modo inductivo, lo que nos llevó a producir una organización del metatexto para aproximarnos al tercer elemento del ciclo.

La captación del nuevo emergente, como último paso para completar el ciclo, corresponde a la elaboración de uno o varios metatextos analíticos que luego se convertirán en textos interpretativos organizados en torno de elementos aglutinadores que contribuyan a dar respuestas a los objetivos centrales del estudio. Para contribuir con el estudio, se utilizó el software Atlas.ti en su versión 8, como estrategia de organización sistemática de los 37 documentos de las informaciones recolectadas, que generaron, en total, 59 códigos, de los cuales para el presente artículo se utilizó la categoría denominada "usos", compuesta por los códigos: a) usos de las Canaimitas "estudiantes", b) usos de la Canaimita "familia", c) prácticas pedagógicas con Canaimitas y d) tareas, esta última con la subcategoría d.1) tareas con Canaimita que incluyen a la familia.

\section{DELINEANDO EL PROYECTO CANAIMA EDUCATIVO}

La multiplicidad de informaciones disponibles, así como el intercambio de estas, independientemente de la presencia física de las personas, ha generado una adaptación a la forma como los seres humanos utilizan los espacios públicos, la forma de organizarse, nuevas maneras de crear; también han cambiado las formas de compartir o de trabajar (Castells, 1999). En el contexto actual, con un panorama lleno de posibilidades 
para acceder a la información y al conocimiento que es producido por la humanidad, desde cualquier rincón del planeta, mediante las tecnologías digitales, se espera que los sistemas educativos respondan a esta necesidad con cambios adaptativos pertinentes.

La educación es establecida como un derecho fundamental y. con el propósito de que esta pueda realizarse en igualdad de oportunidades para todos los ciudadanos, un gran número de países se han fijado como meta incluir las tecnologías digitales dentro de sus políticas públicas, especialmente las educativas. De ahí que el ingreso de las tecnologías digitales a la escuela está estrechamente vinculado a diversas necesidades, como las exigencias de una sociedad cambiante para adaptarse a una contemporaneidad digital, o también sustentado en asuntos económicos.

En América Latina y el Caribe se han implementado múltiples programas para introducir tecnologías digitales a los espacios educativos, uno de los más reconocidos es el Modelo Uno a Uno (1:1, 1a1, 1-1). Este hace referencia a la disponibilidad de dispositivos digitales, que en la mayoría de los casos se trata de una computadora portátil personal para cada niño, con material educativo insertado previamente. Aunque en América Latina su presencia es reciente, sus bases ideológicas ya poseen aproximadamente más de cuarenta años, bajo la filosofía de Seymour Papert y su concepción del papel que debe cumplir la escuela contemporánea, en la que las computadoras van a ocupar un espacio tan equivalente a lo que en su momento fue el lápiz o el libro; y esto modificará las relaciones de los niños con la forma de producir y obtener conocimientos (Papert, 1997).

Para Papert, usar tecnología digital desde el espíritu construccionista expande ampliamente el número, así como la riqueza en que los niños pueden estar inmersos para crear $y$, consecuentemente, se torna más factible la idea del aprendizaje activo (Papert, 1997). Algunas de estas y otras ideas fueran materializadas por Nicholas Negroponte, director del Instituto de Tecnología de Massachusetts (Media Lab del MIT, Estados Unidos), donde Papert y Negroponte pudieron trabajar juntos compartiendo ideas. Es así que Negroponte, en el 2005, concretizó el programa One Laptop per Child (OLPC), conocido en los países hispanohablantes como Una Laptop por Niño.

La idea de que las computadoras portátiles van a traer progreso se expandió rápidamente por el mundo. Etiopía, Nicaragua, Grecia, Paraguay, Afganistán, Haití, Madagascar, solo por nombrar algunos países, están en la lista de los que cuentan con los más de tres millones de niños que poseen una computadora 1a1. El programa OLPC tiene una hegemonía en el ámbito mundial. De hecho América Latina y el Caribe no escaparon de esta realidad, que está fuertemente presente en la región, aunque sin una implementación homogénea, según características y necesidades particulares de cada país.

En el cono sur, como líderes del programa OLPC, encontramos, desde el 2007, el Plan Ceibal en Uruguay; en el mismo año se implementó en Brasil Um Computador por 
Aluno (UCA) y, en el 2010, se incorpora Conectar Igualdad en Argentina. La justificación para insertar estos planes y programas con tecnologías digitales en las políticas educativas fue la del progreso social, económico, educativo y, posiblemente, una combinación de estos (Cepal, 2016).

Las críticas al programa OLPC no se hicieron esperar desde que Negroponte cambió el rumbo de lo que había planteado. Primero propuso un sistema operativo libre basado en Linux Ilamado Sugar, pero se terminó implementando Windows 10 en las computadoras, es decir, un producto propiedad de la empresa Microsoft. Es por ello que el gobierno de Portugal en el año 2007 desarolla una alternativa denominada Magalhães, que también hace referencia a computadoras portátiles de bajo costo de producción, ensambladas en el mismo país. De esta manera, Portugal comienza otras alianzas en el ámbito internacional para promover la inclusión de las computadoras portátiles en la educación.

Venezuela, por su parte, en el 2008, estableció un convenio de cooperación con Portugal, denominado "Acuerdo complementario de cooperación económica y energética" (Villalobos, 2011). Suscrito bajo la presidencia de Hugo Chávez Frías, con el primer ministro José Sócrates, de Portugal, el convenio proyectaba dinamizar las transacciones comerciales de ambas naciones. De esa manera se fortalecería la política exterior venezolana, atendiendo a la Constitución de la República Bolivariana de Venezuela, cuyo artículo 153 promueve la integración para el provecho de las naciones. No solo hubo intercambio comercial en alimentos y maquinarias, sino también en el sector tecnológico, mediante el proyecto Magalhães, que desde el 2009 se aplicó en Venezuela adaptado como proyecto Canaima Educativo.

Este es un proyecto con computadoras portátiles, popularmente llamadas Canaimitas, que tienen incorporado material educativo relacionado con el Currículo Nacional Bolivariano, para ser implementado en las instituciones públicas del país. Su objetivo general es "promover la formación integral de los niños y niñas venezolanos(as), mediante el aprendizaje liberador y emancipador apoyado por las Tecnologías de la Información Libres" (Centro Nacional de Tecnologías de Información, 2011). Este proyecto pretendía ser un pilar fundamental para un nuevo modelo educativo con miras a la soberanía tecnológica. Forma parte de un conjunto de políticas públicas en el área educativa que intentan dar sustento a los planes estratégicos del Estado, los cuales subrayan la importancia de priorizar los derechos educativos. Se le dio el nombre de Canaima en referencia a varios elementos: en principio es un vocablo de una lengua indígena venezolana perteneciente a la etnia Pemón y es el nombre de un reconocido parque nacional venezolano, ubicado en el estado Bolívar, donde vivía dicha comunidad aborigen.

El proyecto se desarrolló progresivamente en cuatro etapas: Canaima educativo escolar, Canaima educativo va a mi casa, Canaima educativo va a los liceos -este fue nuestro foco de investigación-, y posteriormente Canaima universitario. El proyecto 
planteó en su propuesta tres ámbitos de acción: estudiantes, docentes y familia. Nuestro núcleo de pesquisa fue la familia. Para ello la política educativa estableció objetivos concretos: a) formar a madres, padres y representantes de los niños de educación primaria en el manejo y uso responsable de las TIL ${ }^{4}$, b) fortalecer a la familia en el rol de corresponsables de la educación, a través del uso pedagógico del computador portátil Canaima Educativo, c) orientar a todos los integrantes de la familia en aspectos legales, sociales y promover las organizaciones socioproductivas, d) promover la creación de redes locales, municipales y regionales para favorecer el desarrollo de los contenidos contextualizados, la divulgación, el intercambio de experiencias significativas del uso de las TIL (Venezuela, 2009).

Se emitieron documentos oficiales con una serie de recomendaciones para el uso de la Canaimita, principalmente sobre la constitución de colectivos de profesores y su formación permanente, para que estos sean los encargados, desde su accionar pedagógico, de fortalecer la corresponsabilidad de la familia, así como de la comunidad en la educación de los estudiantes. Se buscaba trascender el aula y hacer uso de otros espacios mediante las Canaimitas. Según los entes responsables, la incorporación de estas "supone el mayor acceso y apropiación de la información" (Venezuela, 2009) por parte de alumnos y docentes.

Sin embargo, cuando nos sumergimos en el campo para recolectar información al respecto percibimos que, al menos en las dos unidades educativas que fueron parte de la investigación, el proceso de integración, formación y acompañamiento planteado en la política educativa no alcanzó sus objetivos. Para ello, es necesario comprender de cuáles familias estamos hablando y el contexto de cultura digital donde estas se desenvuelven.

\section{FAMILIAS, TECNOLOGÍAS DIGITALES Y POLÍTICAS PÚBLICAS}

La familia, como dimensión de análisis, es amplia, debido a que no existe un modelo único de este conjunto de personas y sus formas de organizarse. Observamos que desde sus inicios ha intentado adaptarse al espacio así como al tiempo, ha ajustado su organización según sus necesidades e intereses, integrando las particularidades de cada uno de sus miembros, que, indudablemente, van a estar influenciados por dinámicas de lo global y de lo local, marcando formas propias de vida.

Por tanto, utilizamos el término en plural, "las familias", ya que estas se han reconfigurado y continuarán configurando constantemente a través del tiempo, como lo expresa Castells (1999) "[...] no es que surge un nuevo tipo prevaleciente de familia: la diversidad es la regla" (p. 254). De hecho, las familias participantes en esta investigación se

4 En Venezuela se utilizan las siglas TIL para hacer referencia a las tecnologías de información libres. 
caracterizaron por su naturaleza diversa, la variación de su estructura, temporalidad de las uniones y singularidad de los roles que desempeñan sus miembros, en tanto padres, madres, hijos u otros parientes.

La revolución tecnológica también vino a colaborar para que se comenzara a constituir una mayor diversidad de formas de comprender a las familias, una vez que la manera de organizarse, de comunicarse, de pensar y de actuar ya nunca será igual que antes. Un contexto de aceleración constante, además de la cotidianidad con las tecnologías digitales, las informaciones como articuladoras de las actividades humanas (Lemos, 2015), posibilitan las mudanzas de algunas rutinas en casa. La mezcla con elementos de otras eras culturales implicó y continuará implicando un reajuste social (Santaella, 2003) y esto abrió paso a generar otra cultura contemporánea en el ámbito familiar.

En la contemporaneidad vivimos nuestro cotidiano digitalizado y nuestros hogares y los miembros de las familias no escapan de esta realidad. Si observamos a nuestro alrededor, muchos de los procesos de la actualidad son facilitados por medio de dispositivos digitales, su evolución así como la rapidez de hoy hacen necesario que estos muden, se adapten, además reconfiguren sus características, para atender nuestras exigencias y así permitir movilizarnos con ellos. De esta manera, volvimos a los dispositivos digitales más portables para cubrir varias necesidades, los hicimos más híbridos, para cumplir con varias funciones, les permitimos conectarse a la red y cada vez los miniaturizamos aún más para facilitar nuestros desplazamientos por todos los espacios. Entonces, podemos subrayar que la principal característica de las tecnologías digitales, hoy, es la movilidad (Lemos, 2009).

Aunado a ello la llegada del internet potencializó la formación de otra estructura comunicativa y organizativa basada en redes, "la sociedad en red" (Castells, 2004), donde existe un constante flujo de corrientes de informaciones, donde se redefinieron los usos de las tecnologías digitales y se desarrollaron otros modos de generar conocimiento. Si a esto le agregamos el desenvolvimiento de la conexión inalámbrica, Wireless, o comúnmente conocida como wifi, entenderemos que todo ello impulsó el desarrollo de nuevas prácticas sociales. De ahí que la emergencia de ese sistema dinámico propició una mudanza en las formas de ser, relacionarnos con el saber y con el mundo a través del compartir, socializar, construir colectivamente, crear, reinventar, es decir, se constituyó un conjunto de prácticas con otras lógicas basadas en la cultura digital, abarcando principalmente la comunicación, las redes sociales y la forma de producir conocimiento (Castells, 2004; Jenkins, 2008; Lévy, 2007).

Pretto y Silveira (2008) entienden la cultura contemporánea como la cultura digital. Cuando nos referimos a esta, pretendemos aproximarnos a una definición amplia, permeable, abierta, sin ánimos de amurallar un término que se encuentra dentro de contextos mutables; además, no la limitamos al producto resultante del uso exclusivo 
de dispositivos tecnológicos digitales o el acceso al internet, más bien, es un vínculo sociocultural que emerge a partir de la interrelación entre la sociedad, la cultura y las tecnologías. Por tanto, la cultura digital es un conjunto de prácticas sociales con tecnologías digitales, en un ambiente ya sea online u offline, donde se desarrollan procesos comunicativos e interactivos horizontalizados, para lo cual es fundamental la creación colaborativa y la socialización de conocimientos (Bonilla y Pretto, 2015; Costa, 2008; Sampaio y Bonilla, 2012; Lévy, 2007).

Es así que cuando hablamos de lo digital como parte de la cultura contemporánea, como lo expone Costa (2008), nos remitimos a elementos que van más allá de la esfera tecnológica o a las causas, consecuencias o posibilidades que ha alcanzado la evolución de las tecnologías digitales. Es necesario enfatizar que las tecnologías digitales también engloban otras formas de pensar, organizarse y actuar. Una familia en el contexto digital, o "familia digital", como la denomina Montoya, Castro y Bonilla (2018), no solo cuenta con dispositivos digitales en sus casas o los utiliza, sino que reconoce el potencial de las tecnologías digitales, se apropia de ellas, crea otros conocimientos, las usa para establecer diálogos constantes, así como para trabajar colaborativamente entre los miembros, además, está en la capacidad de crear redes para el intercambio de saberes, logrando fijar soluciones conjuntas frente a los desafíos dentro o fuera del hogar.

Sin embargo, para hablar de familias digitales en el contexto venezolano con la incorporación de las Canaimitas a los hogares, es imprescindible establecer la formación como la característica esencial para el fortalecimiento de estas prácticas capaces de reconfigurarse junto a las familias. Que no se suponga que apenas con la introducción de dispositivos de lenguaje digital en su cotidianidad esto acontecerá de manera inmediata. Más bien, es necesario que los objetivos apunten a la obtención de conocimiento, así como a la practicidad en la vida cotidiana y la generación de nuevos saberes.

Para ello es esencial comprender que las tecnologías digitales no son simples "herramientas", sino que trascienden hasta llegar a una verdadera apropiación, permitiendo ser estructurantes de las prácticas cotidianas (Pretto y Silveira, 2008) dentro y fuera del hogar. En este sentido, el Estado a través de las intervenciones públicas en las realidades educativas no debe limitarse apenas a dirección de políticas, es necesario que estas sean contextualizadas, reconfiguradas, integradas a la realidad y a su vez integradoras (Montoya González, 2018), con la posibilidad de que se puedan ser redireccionadas, mudando antiguas acciones insatisfactorias.

Tanto las familias, como las políticas públicas, son instituciones que cumplen un rol preponderante en nuestro sistema ya que ambas condicionan la vida de los sujetos sociales, por ello es preciso adicionar otras piezas fundamentales a la hora de establecer políticas educativas con foco en las familias. Entre esos elementos que se deben tener en cuenta para establecerlas con efectividad están los procesos de globalización, los 
cambios demográficos, la desigualdad económica (no solo con relación a otros países, sino también dentro del mismo país), los movimientos migratorios, el sistema de creencias religiosas, los avances en tecnologías, comunicaciones y las capacidades del propio Estado para acompañar los proyectos implementados.

Además, pensar en políticas públicas no solo para las familias, sino con un foco integrado, es decir, pensar junto con las familias, ya que debe existir una implicación, un conocimiento mayor de cómo estas se presentan hoy, derivado de las demandas en cuanto a sus necesidades sociales. En algunos casos, las políticas son pensadas y ejecutadas para familias que no van de la mano con la realidad actual, es decir, son políticas hechas para modelos familiares antiguos. Tampoco son ejecutadas para cubrir sus necesidades, lo que trae como consecuencia que las familias y las políticas públicas tomen caminos divergentes. Entonces, el diagnóstico de lo que sucede en la realidad es básico y trascendental para pensar en acciones que se puedan ejercer no en la familia, más bien junto a estas.

Si dirigimos la mirada para América Latina, podemos observar que no escapa de la realidad que venimos planteando, debido a que los estudios con las familias y políticas públicas educativas que integran tecnologías digitales siguen siendo incipientes; estas se han implementado desde referencias de políticas globales. América Latina no es una región homogénea, por lo que no puede ni debe ser estandarizada. En los diferentes proyectos presentados en América Latina que incluyen tecnologías digitales móviles, especialmente los de modelos 1a1, como Plan Ceibal, Um Computador por Aluno y Conectar Igualdad, los procesos se mantuvieron con algunas variaciones, pero en la mayoría emana la categoría "inclusión social" como eje central. Estos proyectos tomaron como desafío las instituciones escolares para ser puerta de entrada de las políticas de incorporación de tecnologías digitales y de esta manera acceder a las casas y a las familias.

Asimismo, las políticas educativas del Estado venezolano han introducido proyectos y programas para que la población venezolana, desde temprana edad, entre en contacto con las tecnologías digitales, como es el caso del proyecto Canaima Educativo, con la finalidad de que las computadoras llegaran a los espacios educativos de la población con menos poder adquisitivo. Este proyecto tampoco escapó de la complejidad, de los imprevistos que surgen en el proceso. Aunque es claro que "todo proyecto TIC es complicado y no se debe esperar que ninguno funcione fluidamente" (Warschauer y Niiya, 2014, p. 18), se hace necesario pensar de forma conjunta, con el fin de abordar lo más ampliamente posible los detalles planificados y no planificados, porque una política en educación que no esté articulada con acciones de otras políticas públicas, desde su nacimiento, está destinada al fracaso. Implementar una política con las familias implica un desafío que radica en todas las articulaciones que esta pueda poseer. 


\section{FAMILIAS: NECESIDADES, DESEOS E INTERESES CREANDO PRÁCTICAS ESPONTÁNEAS EN LA CULTURA DIGITAL}

Para interpretar los usos que las familias atribuyen a la computadora Canaimita, observamos cuando este dispositivo digital portátil llega a las casas sin que haya una estrategia explícita para el desenvolvimiento del proyecto. Vemos que las prácticas cotidianas se desencadenan en un proceso que emerge de manera natural por parte de cada uno de los miembros de las familias participantes en esta investigación.

La dinámica observada en la cotidianidad de nuestras cuatro familias "[...] parte del descubrimiento progresivo y de la familiarización con los modos de operar de la máquina. Hay una práctica que parte de la racionalidad de la máquina y otra, más libre y espontánea, que parte de la imaginación del usuario" (Gómez, 2002, p. 293). Las prácticas espontáneas que las familias están realizando con la computadora portátil Canaima se desencadenan de la imaginación propia de cada uno de los que componen el núcleo familiar, atendiendo principalmente a sus deseos, necesidades e intereses. A partir de estas prácticas, las Canaimitas en el hogar tienen dos vertientes fuertes: la primera es la existencia de una atracción, la cual es experimentada por cada uno de los miembros de la familia; por otra parte, el proceso de consumo se afianza de manera veloz, de manera individualizada y se caracteriza por una personalización de los usos de la tecnología digital (Gómez, 2002).

Principalmente, percibimos que las familias demostraron una reconfiguración frente a las demandas emergentes del contexto de la cultura digital, a través de las actividades que se realizan cotidianamente en el hogar con tecnologías digitales. Fueron prácticas que se desarrollaron de forma espontánea debido a que las tareas que las familias están realizando con la computadora portátil Canaima se desencadenan de la imaginación de cada uno de los que componen el núcleo familiar. Estas prácticas atienden principalmente a sus deseos, necesidades e intereses.

Tomemos algunos ejemplos de las prácticas espontáneas surgidas entre las madres participantes de nuestra investigación. Ellas manifestaron que usaron la Canaima principalmente para revisar el email, y entrar en canales como YouTube. Mara (madre, 53 años, asistente administrativa) dijo:

La usé o la uso todavía para tejer gorros, para tejer paños, para bordar, para transformar los pantalones [...] para inyectar viendo tutoriales... ¿qué más?... ¡Ahhh! para a hacer recetas de cocina y para tratamientos naturales. (Mara, madre, comunicación personal, 25 de julio del 2017)

Otra práctica espontánea interesante fue la que surgió de Tibaire (madre, 45 años, dedicada al hogar), quien explicó que frecuentemente utiliza la Canaimita para colaborar con sus hijos en las tareas. Dio como ejemplo las asignaciones sobre mapas que les solicitan en las aulas de Geografía a sus hijos y ellos le piden que los calque. 
Con los mapas también, porque cada vez que a ellos no les gusta dibujar, entonces yo les decía "háganlo así" ...o sea, yo más o menos, yo veía entonces, le iba haciendo el dibujo [muestra cómo lo hace en la Canaimita]... ¡la utilizo para ayudar a ellos a trabajar! (Tibaire, madre, comunicación personal, 15 de julio del 2017)

El proceso de su práctica espontánea comienza con la búsqueda en internet y luego coloca una hoja de papel en blanco sobre la pantalla de la computadora Canaima, para calcar todos los elementos del mapa. Ella nos mostró cómo lo realiza con mucho cuidado, pues sabe que si lo hace con mucha fuerza dañará la pantalla del dispositivo. Utilizó su imaginación para crear otros modos de hacer, debido a que los recursos económicos de la familia son limitados y dada la situación de crisis en el país durante el 2017. Tibaire encontró una forma de utilizar la computadora desde su imaginación.

Hay personas que saben de sus trabajos con manualidades y le hacen pedidos para diversas ocasiones. Para ella, la manera más práctica, así como económica, de proveerse de los modelos, es copiar los dibujos que necesita directamente de la pantalla de la computadora, debido a los altos costos de las impresiones. Además de los trabajos que realiza dentro y fuera del hogar con la Canaimita, Tibaire explicó que le gusta ver los tutoriales de cocina así como los de manualidades, que le dan nuevas ideas para sus trabajos de decoración. Igualmente expresa que, desde su visión, la computadora la ayudó a comunicarse con otras personas y que lo hace a través de la red social Facebook.

Los dos padres participantes en la investigación, Aramaipuro (padre, 47 años, técnico superior en Computación) y Guaicaipuro (padre, 54 años, ingeniero, abogado y magíster en Economía), manifestaron que sienten una cierta "comodidad" con las tecnologías digitales debido a que tienen estudios relacionados con campos próximos y también tienen trabajos en los cuales utilizan diferentes tecnologías diariamente.

Ambos expresaron que el uso que le otorgaron a la Canaimita cuando esta llegó a casa fue principalmente para explorar la estructura interna, conocer el software, ver las similitudes y diferencias con el software privativo que ellos usualmente utilizan, además de saber las aplicaciones que contenía, es decir, toda la lógica de funcionamiento de la máquina. Lo que percibimos es que predominó la exploración para adquirir conocimiento, más que por la necesidad de uso, el propósito fue ayudar a sus hijos. Es decir, los padres participantes se ocuparon de la labor formativa para los estudiantes y otros miembros de la familia.

Yo creo que lo positivo es que fue un proyecto asequible a todos los estudiantes o a la mayoría de los estudiantes que les llegó, y la parte negativa, como lo decía, requiere, por ejemplo, de un manual de usuario para el uso, para que no tenga tantas restricciones y recomendaciones y por supuesto las asesorías técnicas, que es de lo que más se queja la gente, hay mucha gente que simplemente la lleva y 
paga, porque algo se le dañó y no se la pudieron reparar, no le dieron la atención adecuada. En mi caso porque soy técnico y sé algo. (Aramaipuro, comunicación personal, 15 de julio del 2017)

Ellos suplieron la carencia que existía de un acompañamiento por parte de técnicos especialistas del proyecto, por lo que cumplieron con la función de técnicos en reparación, actualización, tanto de software como de hardware. Estos fueron elementos que reflejaron algunas fragilidades en la operatividad del proyecto Canaima Educativo, en el nivel formativo de los miembros de la familias y de los profesores.

Para la familia, podrían colocar tutoriales de cómo utilizar cada tipo de programa que está allí, por ejemplo Blender. Ahí no aparecía ninguna información de modelados de imágenes 3D y todo lo demás, o sea, si no hay una persona que nos está explicando, alguien, para qué sirve, no van a saber cómo utilizarlo y en teoría lo tienen ahí, pero lo van obviar. (Caricuao, estudiante, comunicación personal, 25 de julio del 2017)

Cada liceo debería de dar como una especie de taller para los profesores para que aprendan a utilizar las Canaimas y ese tipo de dispositivos, para que al momento de utilizarla no se le haga muy complicado y sepan cómo explicarle a los estudiantes, como, qué es lo que van a hacer sus proyectos. (Arichuna, estudiante, comunicación personal, 15 de julio del 2017)

Ahora bien, los cuatro jóvenes estudiantes están marcados por la vivencia en la cultura digital, tienen en sus manos dispositivos digitales portátiles que les fueron entregados en sus instituciones educativas. Ellos han crecido bajo una época digitalizada, lo que es fácilmente observable, no solo por la computadora del proyecto Canaima. Pudimos comprobar que en su cotidianidad manejan diversos dispositivos, smartphone, tabletas digitales, otras computadoras, descargan aplicaciones, las usan, las borran y siempre están en busca de nuevas opciones. Son multitareas. Percibimos que pueden conversar, mandar mensajes por el teléfono, al mismo tiempo que descargan una película del computador de escritorio, escuchan música en la Canaima y hacen investigaciones como parte de una tarea.

Los usos que los estudiantes les asignaron a la computadora Canaimita fueron bastante diversos. Por ejemplo, Arichuna (hijo, 15 años, estudiante de tercer año de educación media) utiliza el dispositivo, como él mismo lo indica, "para todo", desde realizar tareas, divertirse con juegos, hasta estudiar a través de tutoriales en YouTube. De hecho, nos mostró que está haciendo estudios de forma autodidacta sobre tecnologías digitales, específicamente de cómo producir contenidos para YouTube, por lo que abrió un canal y sube "contenidos variados". Nos comenta que pasa muchas horas en la plataforma viendo videos, tutoriales, aprende a editar, colocar sonidos, imágenes y todo lo que se le ocurra. Para él, el youtuber crea, comparte e intercambia conocimientos e informaciones de forma interactiva y libre con otras personas. La Canaimita también posibilitó que los 
chicos se hicieran cada vez más autónomos, que fueran autores, otra forma de ser y de producir conocimiento a través de la creación de nuevos contenidos y remezclando los existentes (Bonilla y Pretto, 2015; Castells, 2004; Jenkins, 2008; Lévy, 2007).

La estudiante Caribay (hija, 17 años, estudiante del quinto año de educación diversificada) menciona que suele utilizar las tecnologías digitales, la Canaimita, así como su smartphone. Las características de portabilidad y tamaño le permiten su transporte dentro y fuera de casa con mayor facilidad. El uso que Caribay le da a la Canaima está fuertemente marcado por las redes sociales, para buscar información de temas que le interesan, subir contenidos de su interés y comunicarse con sus familiares, especialmente con su grupo de pares. "Para buscar la información, hacer trabajos... Para el internet y meterme en las redes sociales y para los juegos". (Caribay, estudiante, comunicación personal, 9 de julio del 2017)

La conexión a través de las redes sociales digitales es, sin duda, la actividad frecuente que se desprende de las conversaciones con los más jóvenes de esta investigación. En la cultura digital, nuevas formas de ser florecen, los jóvenes muestran sus identidades, sus gustos, discuten temas, usan su propio lenguaje. Estar conectado también permite otra forma de socializar en el ciberespacio.

Otro de los estudiantes, Caricuao (hijo, 17 años, estudiante del quinto año de educación diversificada), nos comentó que es youtuber y que sus comienzos los hizo con la Canaimita. Su actividad se desencadena por curiosidad y porque el contexto educativo se lo demandó al entregarle una computadora sin ninguna formación específica. A través de sus estudios espontáneos en casa, aprendió varias técnicas para aplicarlas en las tareas que le asignaban cotidianamente. Refiere que uno de sus profesores les mandaba a hacer videos de un contenido específico de la cátedra en forma de noticiero y debía entrevistar a personas que tuvieran conocimiento del tema. Pero, esa actividad, como muchas otras -y no solo de este profesor, sino de varios-, carecía de sugerencias de cómo hacerlo. Simplemente se le indicaba que la actividad fuera grupal y realizada con la computadora Canaima. Esto dio paso a que en repetidas ocasiones Caricuao tomara el liderazgo.

Durante la investigación, nos comentó que el director de la institución donde él estudia, al enterarse de sus habilidades, le pidió realizar algunos talleres y charlas sobre la Canaimita, con la finalidad de lograr un efecto multiplicador de sus conocimientos. Con ayuda de algunos amigos y de sus hermanos, comenzó a planificar y realizar las actividades solicitadas.

Recuerdo que yo tuve que utilizar mi Canaima para dar explicaciones en el Liceo. También utilicé la Canaima del profesor Catia para dar una explicación completa, sobre cuáles son los usos que se le pueden dar... que, me imagino, que quienes deberían estar promoviendo eso son las personas que las entregan [las Canaimitas] 
tratando de explicarles a todas las personas para que no se les sea tan complicado y no vieran el computador como algo... hummm es difícil de utilizar, esto no sé para qué es, solo entraré en Facebook y ya, lo cerraré y lo apagaré, sino que empezaran a aprender otras cosas que tiene. (Caricuao, estudiante, comunicación personal, 25 de julio del 2017)

Entonces, percibimos que Caricuao se encuentra sumergido en las prácticas propias de la cultura digital, ya que él produce contenidos como youtuber e interactúa en el ciberespacio con sus seguidores, que en muchas ocasiones también son sus compañeros de clases. De esta forma comparten conocimientos. También quedó demostrado que los conocimientos no solo se producen desde dentro de las aulas, en muchas ocasiones los estudiantes llegan cargados de saberes. Observamos que Caricuao lleva a la escuela conocimientos que aprendió fuera de esta, lo que influye en las prácticas escolares. Se rompe la creencia de que el conocimiento tiene una sola dirección, escuela-casa, y se muestra que también puede darse en el sentido casa-escuela, es decir, el conocimiento se alimenta de manera bidireccional.

En ese sentido, las instituciones educativas, como escenario de constantes interacciones y como espacio de socialización por excelencia, no deben permanecer inmóviles ante los impulsos de una sociedad compleja, así como altamente cambiante. A estas se las responsabiliza de la integración de las tecnologías digitales y constantemente están envueltas en desafíos, ya sea por la implementación de políticas públicas o cuando las tecnologías digitales llegan del exterior de forma natural, por las manos de los estudiantes. La escuela también debe ser un eje que articule y que propicie espacios para que elementos como participación, colaboración y creación de saberes, propios de la cultura digital, puedan generarse para alcanzar la sostenibilidad de proyectos, como Canaima Educativo.

\section{REFLEXIONES Y APROXIMACIONES FINALES}

Las tecnologías digitales móviles adquirieron interés en las políticas públicas del Estado venezolano, las cuales se vieron materializadas a través del proyecto Canaima Educativo, con el objetivo de incorporar computadoras portátiles con contenido educativo, no solo en las instituciones de toda la red pública del sistema educativo venezolano, sino que, además, se pretendió introducirlas a los hogares por medio de los estudiantes, a partir de la fase Canaima va a la casa.

A pesar de que existen, desde la política, unos objetivos para desarrollar acciones desde el ámbito familiar y vincular la familia con la escuela, con el propósito de que ambas trabajen en conjunto, ya que se daba por sentado su corresponsabilidad en la educación de los jóvenes, desde nuestra investigación y de acuerdo con el grupo estudiado, percibimos que la política no fue lo suficientemente planificada para abordar otros espacios de forma integral. 
Observamos que las Canaimitas llegan a los hogares de las familias participantes sin un estudio previo y específico, como se reflejó en las conversaciones con los participantes, que incidieron en las carencias desde la entrega de la computadora. Es imprescindible que las políticas públicas en el área educativa sean integrales y al mismo tiempo tengan la capacidad de incorporar otras políticas. La planificación y la integralidad debieron ser elementos esenciales, para que no solo estuviera inmersa el área educativa, sino un equipo multidisciplinario que constantemente estuviera en la disposición de reconfigurar el proyecto desde una política educativa descentralizada, capaz de comprender, atender y reorganizar el proyecto Canaima Educativo de acuerdo con los diversos contextos familiares.

Como consecuencia de la emergencia y convergencia de estos elementos, se desencadena la forma en que cada uno de los miembros de las familias hizo uso y creó otras prácticas con la computadora. Es decir, las familias adaptaron la Canaimita a sus necesidades, intereses, así como sus deseos, desde su cotidianidad, lo que generó prácticas espontáneas por medio de una familiarización que se tornó progresiva en el tiempo y un autoaprendizaje en la forma de utilizar la Canaimita para sustituir sus necesidades. Los modos de interactuar con la Canaimita nacieron desde la curiosidad y la creatividad de nuestros sujetos. Se evidenció que apenas hubo una utilización para el consumo de informaciones y que fue baja la producción de materiales.

A partir de las prácticas cotidianas, percibimos un uso variado de la portátil desde cada una de las generaciones participantes en la investigación. Por parte de las madres, se encuentra el uso del e-mail, las redes sociales como medio de comunicación, además de videos tutoriales, por ejemplo, para hacer costuras, y otras prácticas más curiosas, como calcar los dibujos con una hoja blanca sobre la pantalla de la Canaimita, a semejanza de un tablero de luz.

Los padres participantes se mostraron más familiarizados con las tecnologías digitales, debido a sus estudios académicos en áreas afines. Son los que menos utilizan la Canaimita en casa, aunque en un primer momento su acercamiento fue aproximarse a las portátiles, desde el punto de vista técnico. Es decir, utilizaron la Canaima para conocer nuevos elementos técnicos de hardware y software, acompañar a sus hijos en el proceso de exploración, asumiendo el papel de formador y técnico para los miembros familiares.

El uso que le asignaron los jóvenes a la Canaimita no solo fue como apoyo en sus actividades pedagógicas, sino que ellos también se reapropiaron de la computadora portátil según intereses, deseos y necesidades. La utilización estuvo basada principalmente en la comunicación con el mundo por medio de las redes sociales, cambiaron su vida privada al hacerla más pública, pero la razón de esto es que ellos quieren ser más visibles y esto es una posibilidad que se abre en las redes sociales; ellos van construyendo su personalidad en la red, demostrándola sin menoscabo. 
La producción de conocimientos es bastante frágil debido a la falta de formación, de acompañamiento y acceso a otros elementos importantes como el internet, por lo que apenas dos de nuestros estudiantes crean y comparten contenidos, ya que pertenecen a otro tipo de comunicador, los youtubers. Otras dinámicas fueron los juegos online $\mathrm{u}$ offline, que les permiten estar dentro de la cultura digital. Estas lógicas en torno a lo digital están en constante reconfiguración. Lo que principalmente los mueve es la necesidad de interactuar en diferentes espacios como una especie de combustible diario, que los hace sentirse parte de la contemporaneidad. Esto es perceptible ya que los estudiantes participantes estaban en muchas actividades de forma simultánea, sin límites de fronteras geográficas o de tiempo. Con la ayuda de sus pulgares pueden mandar un mensaje instantáneo, al mismo tiempo, que de la computadora descargan una película. Paralelamente, agarran el teléfono y suben un selfie a otra red social, están en la Canaimita participando de un juego online, y al terminar desarrollan una actividad pedagógica haciendo una presentación para una de sus clases.

Entendemos que estos usos espontáneos, que se desenvuelven en el cotidiano de las familias, se van aproximando a la perspectiva de la cultura digital, ya que se construye, a través de la interacción con la Canaimita, un proceso que no es planificado, pero que constantemente intenta ser reinventado. La portátil se presenta como un elemento desencadenador de mudanzas y de reconfiguraciones de prácticas cotidianas. Esto demuestra que haciendo viables las condiciones adecuadas para que el proyecto trascienda en el tiempo, no solo en cantidad de Canaimitas entregadas, sino en implementación y divulgación de experiencias de aprendizaje desde un enfoque multidisciplinario, participativo, colaborativo, de producción de nuevos conocimientos, tendríamos la formación de sujetos críticos y no apenas consumidores de información, lo que contribuiría a su emancipación y, de esta manera, a la independencia tecnológica del país.

Los elementos que se presentaron durante el desarrollo de este estudio, en cuanto a la formación de los sujetos, la necesidad de un acompañamiento técnico y pedagógico para las familias, así como un programa para la reparación de las computadoras, el reciclaje y la reutilización de las piezas, provocan otros cuestionamientos que se presentan como un desafío para que próximas investigaciones colaboren en los caminos futuros de la reconfiguración del proyecto Canaima Educativo.

\section{REFERENCIAS}

Balcázar, P., González, N., Gurrola, G. y Chimal, A. (2006). Investigación Cualitativa (1. a ed.). Recuperado de http://ri.uaemex.mx/bitstream/handle/20.500.11799/21589/ Investigaci\%F3n\%20cualitativa.pdf?sequence $=3$ 
Bauman, Z. (2003). Modernidad líquida. Recuperado de https://catedraepistemologia.files. wordpress.com/2009/05/modernidad-liquida.pdf

Bonilla, M. H. y Pretto, N. (2015). EmAberto: Movimentos colaborativos, tecnologias digitais e educação. 28(94). Recuperado de http://emaberto.inep.gov.br/index.php/ emaberto/issue/view/196

Capra, F. (1996). A teia da Vida: uma compreensão científica dos sistemas vivos. São Paulo: Cultrix LTDA.

Castells, M. (1999). La era de la información. Economía, sociedad y cultura. El poder de la identidad, Vol. 2. Trad. C. Martínez. Recuperado de https://filosinsentido.files. wordpress.com/2013/06/manuel_castells_la_era_de_la_informacic3b3n_ econobookos-org.pdf

Castells, M. (2004). La era de la información: Economía, sociedad y cultura, Vol. 3. México D.F.: Siglo XXI.

Centro Nacional de Tecnologías de Información. (2011). LaTItud. 7. edición. Recuperado de http://www.cnti.gob.ve/noticias/revista/latitud/file/11-septima-edicion.html

Cepal. (2016). Cepal: El crecimiento sostenible a largo plazo solo se logrará con la digitalización de la economía. Recuperado de http://innovalac.cepal.org/2/es

Costa, R. (2008). A cultura digital (3. ${ }^{\text {a }}$ ed.). São Paulo: PubliFolha.

Gómez, C. (2002). Usos sociales de las tecnologías de la información y comunicación. UAM-X• MÉXIC0. 287-305. Recuperado de http://cmap.upb.edu.co/rid=1Q0C0QTBH-1MJ7 2H0-19C1/usosSocialesTIC_carmenGomez.pdf

Jenkins, H. (2008). Convergence Culture: la cultura de la convergencia de los medios de comunicación. Recuperado de https://stbngtrrz.files.wordpress.com/2012/10/ jenkins-henry-convergence-culture.pdf

Lemos, A. (2009). Cultura da Mobilidade. Revista Famecos. 16(40), 28-35. Recuperado de http://revistaseletronicas.pucrs.br/ojs/index.php/revistafamecos/article/view 16314

Lemos, A. (2015). Cibercultura: tecnologia e vida social na cultura contemporânea (7a ed.). Recuperado de http://www.e-publicacoes.uerj.br/index.php/logos/article/ download/14575/11038

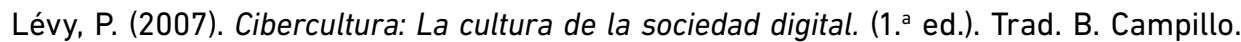
Recuperado de https://antroporecursos.files.wordpress.com/2009/03/levy-p1997-cibercultura.pdf 
Macedo, R. S. A. (2010). Etnopesquisa Crítica, Etnopesquisa-Formação. Brasília: Liber Livro Editora.

Macedo, R. S. , Assis, A., Guerra, D., Rangel, L. y Macedo de Sá, S. M. (Orgs.). (2014). Saberes implicados, saberes que formam: a diferença em perspectiva. Salvador de Bahia: EDUFBA.

Montoya González, Y. D. V. (2018). Proyecto Canaima Educativo y la cultura digital de las familias venezolanas (Dissertação Mestrado em Educação). Universidade Federal da Bahia, Faculdade de Educação, Programa de Pós-Graduação em Educação, Salvador.

Montoya, Y., Castro, C. y Bonilla, M. H. (2018). Más allá del control parental: redefiniendo a la familia digital. Recuperado de http://www.upf.br/_uploads/Conteudo/ senid/2018-artigos-completos/178910.pdf

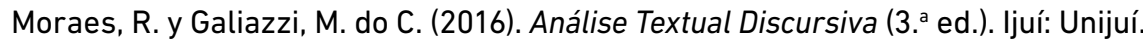

Papert, S. (1997). La familia conectada. Padres, hijos y computadoras. Trad. M. C. Pinto. Recuperado de https://tecnoeducativas.files.wordpress.com/2017/03/papertlafamiliaconectada.pdf

Pretto, N. y Silveira, S. (2008). Além das redes de colaboração: internet, diversidade cultural e tecnologias do poder. Recuperado de https://repositorio.ufba.br/ri/bitstream/ ufba/211/1/Alem\%20das\%20redes\%20de\%20colaboracao.pdf

Sampaio, J. y Bonilla, M. H. (2012). Os jovens na contemporaneidade: a experiência da articulação entre a dinâmica da escola e um projeto de inclusão digital. Anais do SENID. Recuperado de http://gepid.upf.br/senid/2012/anais/95071.pdf

Santaella, L. (2003). Culturas e Artes do Pós-Humano: da Cultura das Mídias à Cibercultura. São Paulo: Paulus Editora.

Venezuela. (2009). Canaima Educativo. Recuperado de http://www.canaimaeducativo.gob. ve/

Villalobos Soto, D. J. (2011). Acuerdo comercial entre Venezuela y Portugal, Caso: acuerdo complementario en el marco de la cooperación económica y energética. Revista Venezolana de Análisis de Coyuntura. XVII, 115-129. Recuperado de saber.ucv.ve/ ojs/index.php/rev_ac/article/download/3239/3101

Warschauer, M. y Niiya, M. (2014). Medios digitales e inclusión social. Revista peruana de investigación educativa, 6(1), 09-32. Recuperado de http://www.siep.org.pe/ wp-content/uploads/06_01_Warschauer.pdf 